\title{
Integrating Network Pharmacology and Experimental Verification to Explore the Mechanism of Effect of Zuojin Pills in Pancreatic Cancer Treatment
}

\author{
Kunpeng Wang' \\ Xiongying $\mathrm{Miao}^{2}$ \\ Fanhua Kong ${ }^{2}$ \\ Siqi Huang $\mathbb{D}^{3}$ \\ Jinggang Mo' \\ Chong Jin' \\ Yanwen Zheng ${ }^{2}$ \\ 'Department of General Surgery, Taizhou \\ Central Hospital (Taizhou University \\ Hospital), Taizhou, 318000 , Zhejiang, \\ People's Republic of China; ${ }^{2}$ Department \\ of General Surgery, The Second Xiangya \\ Hospital, Central South University, \\ Changsha, 4I00II, Hunan, People's \\ Republic of China; ${ }^{3}$ Department of \\ Integrated Traditional Chinese \& \\ Western Medicine, The Second Xiangya \\ Hospital, Central South University, \\ Changsha, 4I00II, Hunan, People's \\ Republic of China
}

Correspondence: Yanwen Zheng Department of General Surgery, The Second Xiangya Hospital, Central South University, Changsha, 4I00II, Hunan, People's Republic of China Email zyw.1018@csu.edu.cn
Background and Aim: Pancreatic cancer is one of the most malignant tumors worldwide. Zuojin pills (ZJP), a traditional Chinese medicine (TCM) formula, which can treat a variety of cancers. However, the active compounds present in ZJP and the potential mechanisms through which ZJP acts against pancreatic cancer have not been thoroughly investigated.

Methods: Data on pancreatic cancer-related genes, bioactive compounds, and potential targets of ZJP were downloaded from public databases. Bioinformatics analysis, including protein-protein interaction (PPI), Gene Ontology (GO), and Kyoto Encyclopedia of Genes and Genomes (KEGG) analyses, was conducted to identify important components, potential targets, and signaling pathways through which ZJP affects pancreatic cancer. The results of this analysis were verified by in vitro experiments.

Results: The network pharmacology analysis results showed that 41 compounds and 130 putative target genes of ZJP were associated with anti-pancreatic cancer effects. ZJP may exert its inhibitory effects against pancreatic cancer by acting on key targets such as JUN, TP53, and MAPK1. Moreover, KEGG analysis indicated that the anti-pancreatic cancer effect of ZJP was mediated by multiple pathways, such as the PI3K-AKT, IL-17, TNF, HIF-1, and P53 signaling pathways. Among these, the PI3K-AKT signaling pathway, which included the highest number of enriched genes, may play a more important role in treating pancreatic cancer. The in vitro results showed that ZJP significantly inhibits the cell cycle and cell proliferation through the $\mathrm{PI} 3 \mathrm{~K} / \mathrm{AKT} /$ caspase pathway and that it can induce apoptosis of pancreatic cancer cells, consistent with the results predicted by network pharmacological methods.

Conclusion: This study preliminarily investigated the pharmacological effects of ZJP, which appear to be mediated by multiple compounds, targets and pathways, and its potential therapeutic effect on pancreatic cancer. Importantly, our work provides a promising approach for the identification of compounds in TCM and the characterization of therapeutic mechanisms.

Keywords: pancreatic cancer, Zuojin pill, network pharmacology, traditional Chinese medicine, proliferation, apoptosis

\section{Introduction}

Pancreatic cancer is one of the deadliest tumors, and its incidence has increased in recent years. ${ }^{1-4}$ According to GLOBOCAN 2020 of the International Agency for Research on Cancer, 495,773 new cases of pancreatic cancer were reported in 2020 was, accounting for $2.6 \%$ of the total cancer incidence. The number of new deaths was 466,003 , accounting for $4.7 \%$ of the total cancer mortality. ${ }^{5}$ Even with surgery and standard chemotherapy, most patients eventually relapse, and the 5-year survival rate is only $2-9 \%{ }^{6,7}$ In the past decade, patient outcomes have improved 
dramatically with advances in diagnostic techniques, perioperative therapy, radiotherapy and treatment for advanced pancreatic cancer. ${ }^{8,9}$ Although the survival rates for pancreatic cancer patients have improved, it remains one of the deadliest malignancies. To make matters worse, most patients are already in an advanced stage of the disease or have distant metastases by the time they are definitively diagnosed. ${ }^{10}$ Thus, it is of great significance to explore new therapeutic methods and therapeutic targets for improving the survival of pancreatic cancer patients.

TCM has been used clinically for more than 2000 years. It has become the most common complementary and alternative therapy strategy in China and neighboring countries and has gradually become accepted due to its remarkable efficacy, the wide variety of drug sources it offers, and its low toxicity. ${ }^{11}$ Many TCM compounds have been used to treat tumors or as adjuvants in routine chemotherapy. ${ }^{12,13}$ In addition, TCM can improve therapeutic outcomes, reduce the incidence of adverse reactions, improve patients' quality of life and prolong survival in combination with chemotherapy or radiotherapy. ${ }^{14-16}$ Due to the above advantages it offers, TCM is becoming increasingly popular in Western countries. ${ }^{17,18}$

Zuojin pill (ZJP) is a TCM compound that has been recorded in the Chinese Pharmacopoeia Guidelines for the Treatment of Gastrointestinal Diseases (Chinese Pharmacopoeia Committee, 2015) and is the source of a drug pair commonly used in TCM. ZJP has only two components: Rhizoma Coptidis (RC) and Evodiae Fructus (EF). ${ }^{19,20} \mathrm{RC}$ and EF were prepared in a 6:1 (W/W) ratio. RC is extracted from Coptis chinensis and has been widely used to treat diseases of the digestive tract, ${ }^{21}$ liver damage, diabetes and other diseases. ${ }^{22,23} \mathrm{EF}$, the immature fruit of Evodia fructus, is widely used to treat headache, inflammation, hypertension and other conditions. ${ }^{24}$ Previous studies have shown that ZJP and the Chinese herbal medicines derived from it, $\mathrm{RC}$ and $\mathrm{EF}$, and their active compounds exhibit a variety of anticancer pharmacological effects through various mechanisms of action. ${ }^{21,25}$ Moreover, a network pharmacological analysis by Guo et al showed that ZJP had a good therapeutic effect on liver cancer, mainly through the regulation of cell proliferation and survival through the EGFR/ MAPK, PI3K/NF- $\mathrm{B}$ and CCND1 signaling pathways. ${ }^{19}$ In addition, ZJP significantly inhibited the proliferation of human tumor cell lines by inducing mitochondrial apoptosis and had significant inhibitory effects on HepG2-derived mouse tumors, breast cancer, colorectal cancer, and other cancers. $^{25-27}$
In recent years, biotechnology has made great progress. Network pharmacology based on large databases has become a powerful tool for studying TCM because it allows investigators to study the mechanism of action of compounds from the molecular level to the pathway level. $^{28}$ Network pharmacology studies the relationships among drugs, targets and diseases by revealing the actions and therapeutic mechanisms of compounds used in TCM and intuitively illustrates the mechanisms of action involved in drug-target-disease networks. ${ }^{29}$ Moreover, network pharmacology embodies the concept of the holistic philosophy of TCM. ${ }^{30}$ As a new technology, this method upgrades the current "one target-one drug" model to a new network-based multitarget approach that is helpful for evaluating the rationality and compatibility of TCM applications.

This study aimed to predict and validate the active compounds present in ZJP and their potential protein targets and pathways, as well as the molecular mechanisms involved in the action of these compounds on pancreatic cancer, by means of network pharmacology and in vitro validation. The detailed technical strategy used in the current study is shown in Figure 1.

\section{Materials and Methods}

\section{Data Preparation}

\section{Compounds and Target Screening}

All ingredients of two herbs, RC and EF, were collected from the Traditional Chinese Medicines for Systems Pharmacology Database and Analysis Platform (TCMSP, available online: http://tcmspw.com/tcmsp.php) and the Bioinformatics Analysis Tool for Molecular mechANism of Traditional Chinese Medicine (BATMAN-TCM, available online: http://bionet.ncpsb.org/batman-tcm/index.php/ home/index/result/jobId/batman-I2019-04-16-177451555420409.html). Oral bioavailability ${ }^{31}$ (OB) and druglikeness (DL), two absorption, distribution, metabolism and excretion (ADME)-related properties, were used as the screening criteria, and only the ingredients that met the $\mathrm{OB} \geq 30 \%$ and $\mathrm{DL} \geq 0.18$ criteria were selected for subsequent research.

The putative targets of the ingredients were acquired from the TCMSP website, which describes drug targets and their relationships to diseases in detail. The putative targets were then annotated with the official gene symbols by UniProt (http://www.UniProt.org/). 


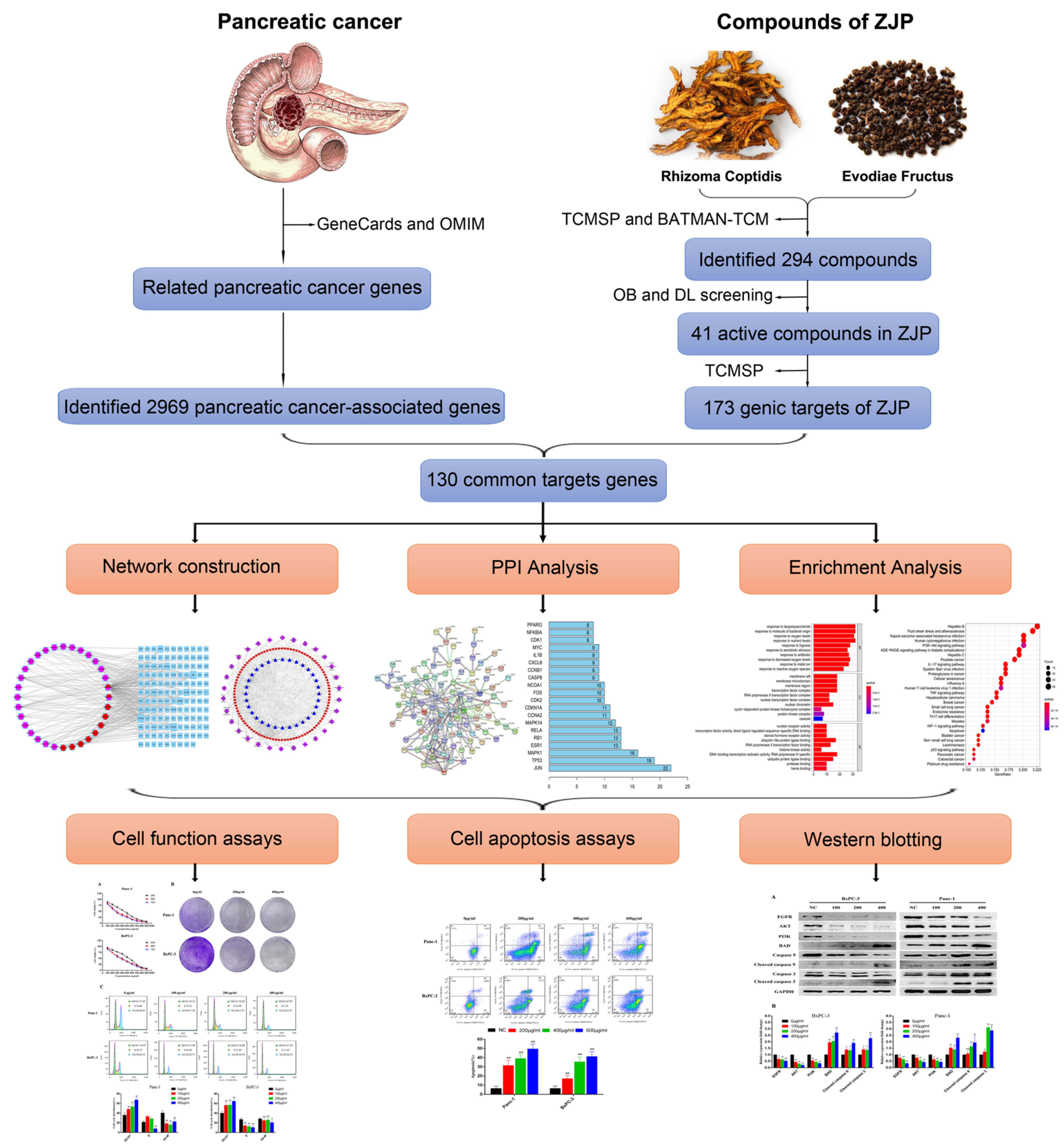

Figure I Technical strategy used in the current study.

\section{Potential Targets of ZJP Against Pancreatic Cancer}

Pancreatic cancer-related targets were obtained from the GeneCards (http://www.genecards.org/) and Online Mendelian Inheritance in Man (OMIM, http://www. omim.org/) databases. GeneCards, which provides a comprehensive database of human genes, is a powerful searchable database that includes human gene data from multiple databases. OMIM, a constantly updated database, focuses on the relationship between human genetic variation and phenotypic traits. Intersecting genes among potential targets of ZJP and pancreatic cancer-related genes were regarded as potential targets of ZJP in the treatment of pancreatic cancer. 


\section{Bioinformatics Analysis}

\section{Protein-Protein Interaction (PPI) Data}

Potential ZJP targets related to the treatment of pancreatic cancer were input into the Search Tool for the Retrieval of Interacting Genes/Proteins (STRING; https://string-db.org/) with the species limited to "Homo sapiens" and to results with a confidence score $>0.950$.

\section{Enrichment Analysis and Construction of Networks}

The potential biological functions and mechanisms of the retrieved targets were explored further using Gene Ontology (GO) and Kyoto Encyclopedia of Genes and Genomes (KEGG) pathway enrichment analyses conducted using the $\mathrm{R}$ package $(\mathrm{p}<0.05)$. Moreover, herb-compound herb-disease -target (C-D-T) networks and compound-pathway-target (C-P-T) networks were constructed and visualized with Cytoscape 3.7.1.

\section{Experimental Validation}

\section{Preparation of ZJP Aqueous Extract}

RC and EF were obtained from the Pharmacy of the Second Xiangya Hospital of Central South University using the methods outlined in the Chinese Pharmacopoeia (2015 edition). A water extract of ZJP was prepared. Sixty grams of RC and $10 \mathrm{~g}$ of EF were soaked in 8 volumes of distilled water for $30 \mathrm{~min}$; the mixture was decocted for $1.5 \mathrm{~h}$ and centrifuged at 10,000 RPM for $30 \mathrm{~min}$, and the resulting supernatant was collected. The extraction procedure was repeated twice, and the supernatants were combined and evaporated to obtain a powder. The dry powder was redissolved in DMSO to a concentration of $200 \mathrm{mg} / \mathrm{mL}$, and the solution was passed through a filter with a $0.22-\mu \mathrm{m}$ pore diameter and stored at $-20{ }^{\circ} \mathrm{C}$ until further use.

\section{Cell Culture}

The human pancreatic cancer cell lines BxPC-3 and Panc-1 (purchased from ZSBIO in China and identified and certified by the same supplier) were selected for subsequent experiments. Panc- 1 and BxPC- 3 cells were maintained in DMEM supplemented with $10 \%$ fetal bovine serum (FBS), $100 \mathrm{U} / \mathrm{mL}$ penicillin, and $100 \mathrm{mg} / \mathrm{mL}$ streptomycin. All cells were cultured in a humidified incubator with $5 \% \mathrm{CO}_{2}$ at $37{ }^{\circ} \mathrm{C}$.

\section{Cell Viability Assay}

Panc-1 and BxPC-3 cells (5000 cells/well) were seeded in 96well plates and incubated for $24 \mathrm{~h}$ at $37^{\circ} \mathrm{C}$ in a humidified incubator with $5 \% \mathrm{CO}_{2}$. After pretreatment with various concentrations of ZJP $(0,100,200,300,400,500,600,700,800$ and $900 \mu \mathrm{g} / \mathrm{mL}$ ) for 24,48 , and $72 \mathrm{~h}$, ten microliters of Cell
Counting Kit-8 (CCK-8) kit reagent (Genview, USA) was used to determine the optical density (OD) at $450 \mathrm{~nm}$ of the solution in each well using a microplate spectrophotometer (Thermo Fisher, USA). All data were normalized to data obtained for control wells that contained no cells and are presented as the mean $\pm \mathrm{SD}$.

\section{Colony Formation Assay}

Panc-1 and BxPC-3 cells were seeded in 6-well plates at 5000 cells per well. After incubation for $24 \mathrm{~h}$, ZJP was added to some wells (Panc-1 cells were treated with 200 and $400 \mu \mathrm{g} / \mathrm{mL}$ ZJP; BxPC-3 cells were treated with 200 and $400 \mu \mathrm{g} / \mathrm{mL}$ ZJP) for 3 consecutive days. After fixation with $4 \%$ paraformaldehyde for $30 \mathrm{~min}$, the cells were stained with crystal violet solution for $2 \mathrm{~h}$, washed with distilled water and photographed manually.

\section{Flow Cytometry for Cell Cycle Analysis}

Panc- 1 and BxPC-3 cells were seeded in 6-well plates at $5 \times 10^{5}$ cells/well. After incubation of the plates for $24 \mathrm{~h}$, ZJP was added to some wells (Panc-1 cells were treated with 100, 200 and $400 \mu \mathrm{g} / \mathrm{mL}$ ZJP; BxPC-3 cells were treated with 100, 200 and $400 \mu \mathrm{g} / \mathrm{mL}$ ZJP) for $48 \mathrm{~h}$. The cells were collected and fixed with $70 \%$ ethanol overnight at $4{ }^{\circ} \mathrm{C}$. After fixation, the cells were stained with propidium iodide (PI, $50 \mu \mathrm{g} / \mathrm{mL}$, Genview, USA) for $45 \mathrm{~min}$ in the dark. The cell samples were assessed with a Canto II flow cytometer (BD Bioscience, USA) for cell cycle analysis.

\section{Flow Cytometry for Analysis of Cell Apoptosis}

Panc-1 and BxPC-3 cells were seeded in 6-well plates at $3 \times 10^{5}$ cells/well. After incubation for $24 \mathrm{~h}$, ZJP was added to some wells (Panc-1 cells were treated with 200, 400 and $600 \mu \mathrm{g} / \mathrm{mL}$ ZJP; BxPC-3 cells were treated with 200,400 and $600 \mu \mathrm{g} / \mathrm{mL}$ ZJP) for $48 \mathrm{~h}$. Cell apoptosis was measured by flow cytometry using an annexin V-fluorescein isothiocyanate/propidium iodide (annexin V-FITC/PI) apoptosis detection kit (Genview, USA) according to the manufacturer's instructions. The cells were collected and washed twice with cold phosphatebuffered saline, resuspended in $500 \mu \mathrm{L} 1 \times$ binding buffer to which $5 \mu \mathrm{L}$ annexin V-FITC and $5 \mu \mathrm{L}$ PI staining solution had been added, and incubated for $15 \mathrm{~min}$ in the dark at room temperature. The sample was then mixed with an addition $500 \mu \mathrm{L}$ of $1 \times$ binding buffer. Finally, cell apoptosis was measured by flow cytometry (BD Bioscience, USA), and the cytometric data were analyzed using FlowJo 7.6 software (De Novo Software, Los Angeles, CA, USA). 


\section{Western Blotting}

Panc- 1 and BxPC-3 cells were seeded in 6-well plates at $3 \times 10^{5}$ cells/well. After incubation for $24 \mathrm{~h}$, the cells were treated with or without ZJP for $48 \mathrm{~h}(100,200$ and $400 \mu \mathrm{g} / \mathrm{mL}$ ZJP for Panc1 and BxPC-3 cells, respectively). The cells were harvested using a micro scraper. Total protein was obtained from the cells in radioimmunoprecipitation assay (RIPA) buffer (Beyotime, P0013B, Shanghai, China) containing phenylmethylsulfonyl fluoride (PMSF), aprotinin, and a phosphatase inhibitor. After centrifugation $\left(14,000 \times \mathrm{g}\right.$ at $4{ }^{\circ} \mathrm{C}$ for $\left.15 \mathrm{~min}\right)$, the supernatants were collected, and the protein concentration was measured using the BCA Protein Assay Kit (Genview, USA). A total of $25 \mu \mathrm{g}$ of each protein sample was separated by SDS-PAGE and transferred to polyvinylidene fluoride (PVDF) membranes (Millipore, Bedford, MA, USA). The membranes were blocked with $5 \%$ bovine serum albumin (BSA) (Genview, USA) for $2 \mathrm{~h}$ at room temperature. Primary antibodies that recognize the following proteins were then added at the indicated dilutions, and the membranes were incubated overnight at $4{ }^{\circ} \mathrm{C}$ : GAPDH $(1: 10,000$, ABclonal), EGFR (1:1000, ABclonal), AKT (1:1000, Cell Signaling Technology), BAD (1:1000, Cell Signaling Technology), PI3K (1:1000, Proteintech), caspase 3 (1:1000, Proteintech), and caspase 9 (1:1000, Proteintech). A secondary antibody (1:5000, ABclonal) was then added, and the membranes were incubated for $2 \mathrm{~h}$ at room temperature. The immunoreactive bands were developed using an enhanced chemiluminescence detection kit (Genview, USA).

\section{Statistical Analysis}

Statistical analysis was performed using Prism 6 software. The data are expressed as the mean $\pm \mathrm{SD}$ and were analyzed using Student's $t$-test. Differences between groups were considered statistically significant if $\mathrm{P}<0.05$.

\section{Results}

\section{Compounds and Putative Targets of ZJP}

A total of 294 ingredients of ZJP were identified (71 in RC and 226 in EF) after a comprehensive search of the TCMSP and BATMAN-TCM databases. According to the screening criteria $(\mathrm{OB} \geq 30 \%$ and $\mathrm{DL} \geq 0.18), 41$ ingredients were included in the subsequent research after eliminating ingredients that were the same as those of RC (ie, berberine, quercetin, and obacunone). The details are presented in Table 1. Overall, 173 putative targets of ZJP were identified through the TCMSP website after marking them with their corresponding gene symbols.

\section{Potential Targets and C-D-T Network Construction}

We comprehensively searched GeneCards and OMIM using "pancreatic cancer" as a keyword and identified a total of 2969 pancreatic cancer-associated genes. After comparing 173 targets of ZJP and 2969 pancreatic cancerassociated genes, 130 common targets were obtained for

Table I Compounds of ZJP

\begin{tabular}{|l|c|l|l|l|c|}
\hline MOL ID & MOL Name & OB & DL & Medicine & Database Source \\
\hline MOL001454 & Berberine & 38.86 & 0.78 & HL, WZY & TCMSP, BATMAN-TCM \\
\hline MOL000098 & Quercetin & 46.43 & 0.28 & HL, WZY & TCMSP \\
\hline MOL013352 & Obacunone & 43.29 & 0.77 & HL, WZY & TCMSP \\
\hline MOL002894 & Berberrubine & 35.74 & 0.73 & HL & TCMSP \\
\hline MOL002903 & (R)-Canadine & 55.37 & 0.77 & HL & TCMSP \\
\hline MOL002897 & Epiberberine & 43.09 & 0.78 & HL & TCMSP \\
\hline MOL002904 & Berlambine & 36.68 & 0.82 & HL & TCMSP \\
\hline MOL002907 & Corchoroside A_qt & 104.95 & 0.78 & HL & TCMSP \\
\hline MOL000622 & Magnograndiolide & 63.71 & 0.19 & HL & TCMSP \\
\hline MOL000762 & Palmidin A & 35.36 & 0.65 & HL & TCMSP \\
\hline MOL000785 & Palmatine & 64.6 & 0.65 & HL & TCMSP \\
\hline
\end{tabular}

(Continued) 
Table I (Continued).

\begin{tabular}{|c|c|c|c|c|c|}
\hline MOL ID & MOL Name & OB & DL & Medicine & Database Source \\
\hline MOL00I458 & Coptisine & 30.67 & 0.86 & $\mathrm{HL}$ & TCMSP \\
\hline MOL002668 & Worenine & 45.83 & 0.87 & $\mathrm{HL}$ & TCMSP \\
\hline MOL008647 & Moupinamide & 86.71 & 0.26 & $\mathrm{HL}$ & TCMSP \\
\hline MOL002662 & Rutaecarpine & 40.3 & 0.6 & WZY & TCMSP, BATMAN-TCM \\
\hline MOL000354 & Isorhamnetin & 49.6 & 0.31 & WZY & TCMSP \\
\hline MOL000358 & Beta-sitosterol & 36.91 & 0.75 & WZY & TCMSP \\
\hline MOL000359 & Sitosterol & 36.91 & 0.75 & WZY & TCMSP \\
\hline MOL003942 & Rutaevine & 66.05 & 0.58 & WZY & TCMSP \\
\hline MOL003943 & Rutalinidine & 40.89 & 0.22 & WZY & TCMSP \\
\hline MOL003947 & I-methy|-2-[(Z)-pentadec-|0-eny|]-4-quinolone & 48.45 & 0.46 & WZY & TCMSP \\
\hline MOL003950 & I-methyl-2-[(Z)-undec-6-enyl]-4-quinolone & 48.48 & 0.27 & WZY & TCMSP \\
\hline MOL003956 & Dihydrorutaecarpine & 42.27 & 0.6 & WZY & TCMSP \\
\hline MOL003957 & I-methyl-2-pentadecyl-4-quinolone & 44.52 & 0.46 & WZY & TCMSP \\
\hline MOL003958 & Evodiamine & 86.02 & 0.64 & WZY & TCMSP \\
\hline MOL003960 & I-(5,7,8-trimethoxy-2,2-dimethylchromen-6-yl)ethanone & 30.39 & 0.18 & WZY & TCMSP \\
\hline MOL003963 & Hydroxyevodiamine & 72.11 & 0.71 & WZY & TCMSP \\
\hline MOL003964 & I-methyl-2-undecyl-4-quinolone & 47.59 & 0.27 & WZY & TCMSP, BATMAN-TCM \\
\hline MOL003972 & I-methyl-2-nonyl-4-quinolone & 48.42 & 0.2 & WZY & TCMSP \\
\hline MOL003974 & Evocarpine & 48.66 & 0.36 & WZY & TCMSP, BATMAN-TCM \\
\hline MOL003975 & Icosa-II, |4, I7-trienoic acid methyl ester & 44.81 & 0.23 & WZY & TCMSP \\
\hline MOL003994 & 24-methyl-3I-norlanost-9(II)-enol & 38 & 0.75 & WZY & TCMSP, BATMAN-TCM \\
\hline MOL004002 & 5alpha-O-(3'-Methylamino-3'-phenylpropionyl) nicotaxine & 30.86 & 0.49 & WZY & TCMSP \\
\hline MOL004004 & 6-OH-Luteolin & 46.93 & 0.28 & WZY & TCMSP \\
\hline MOL0040I4 & Evodiamide & 73.77 & 0.28 & WZY & TCMSP, BATMAN-TCM \\
\hline MOL0040I7 & Fordimine & 55.11 & 0.26 & WZY & TCMSP, BATMAN-TCM \\
\hline MOL0040I8 & Goshuyuamide I & 83.19 & 0.39 & WZY & TCMSP, BATMAN-TCM \\
\hline( & Goshuyuamidell & 69.11 & 0.43 & WZY & TCMSP, BATMAN-TCM \\
\hline MOL004020 & Gossypetin & 35 & 0.31 & WZY & TCMSP, BATMAN-TCM \\
\hline MOL00402I & Gravacridoneshlirine & 63.73 & 0.54 & WZY & TCMSP \\
\hline MOL004025 & $\mathrm{N}$-(2-Methylaminobenzoylt) ryptamine & 56.96 & 0.26 & WZY & TCMSP, BATMAN-TCM \\
\hline
\end{tabular}

further research (Figure 2). The C-D-T network was constructed to reveal the relationship between the two herbs (RC and EF), their compounds and pancreatic cancer (shown in Figure 3). The C-D-T network, which included
162 nodes and 341 edges, clearly demonstrated that ZJP exerts anti-pancreatic cancer effects through multiple compounds and targets. The pink circle represents the compounds present in EF, the red circle represents the 


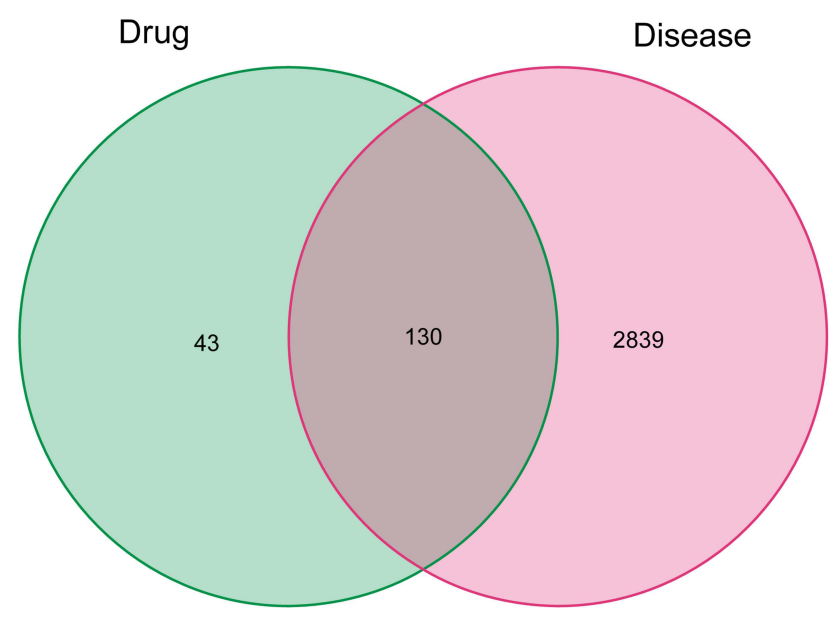

Figure 2 Venn diagram of potential targets of ZJP and pancreatic cancer-related genes.

compounds present in RC, and the pink and red circles represent the compounds (berberine and quercetin) present in both EF and RC. The blue rectangle represents 130 genes: the larger the size of the shape is, the higher is the node degree.

\section{Bioinformatics Analysis PPI Analysis}

The 130 potential targets were submitted to STRING to acquire PPI data that could be used to construct the PPI network, as shown in Figure 4A. After disconnected nodes in the network were hidden, the PPI network consisted of 96 nodes and 227 edges. The nodes and edges represent proteins and protein-protein relationships, respectively. Nodes with many edges were identified as pivotal targets in the network. As shown in Figure 4B, the top 20 proteins with multiple edges in the network were plotted. The results indicate that JUN, TP53, and MAPK1 are probably the most important targets through which ZJP acts on pancreatic cancer.

\section{GO Enrichment Analysis}

GO enrichment analysis was performed to verify the biological functions, including biological processes (BPs), cell components (CCs), and molecular functions (MFs), of the 130 putative target genes that are involved in ZJP's action against pancreatic cancer. A total of 2313 terms were acquired, including 2088 for BP, 59 for CC, and 166 for MF. The main enriched BP terms were response to lipopolysaccharide (GO:0032496), response to molecules of bacterial origin (GO:0002237), and response to oxygen levels. The main enriched $\mathrm{CC}$ terms were membrane raft (GO:0045121), membrane microdomain (GO:0098857), and membrane region (GO:0098589). The top three MF terms were nuclear receptor activity (GO:0004879), transcription factor activity, direct ligand-regulated sequencespecific DNA binding (GO:0098531), and steroid hormone receptor activity (GO:0003707). The top ten $\mathrm{BP}, \mathrm{CC}$, and MF terms ranked according to their q-values are shown in Figure 5A. The redder the color of a term is in the figure, the

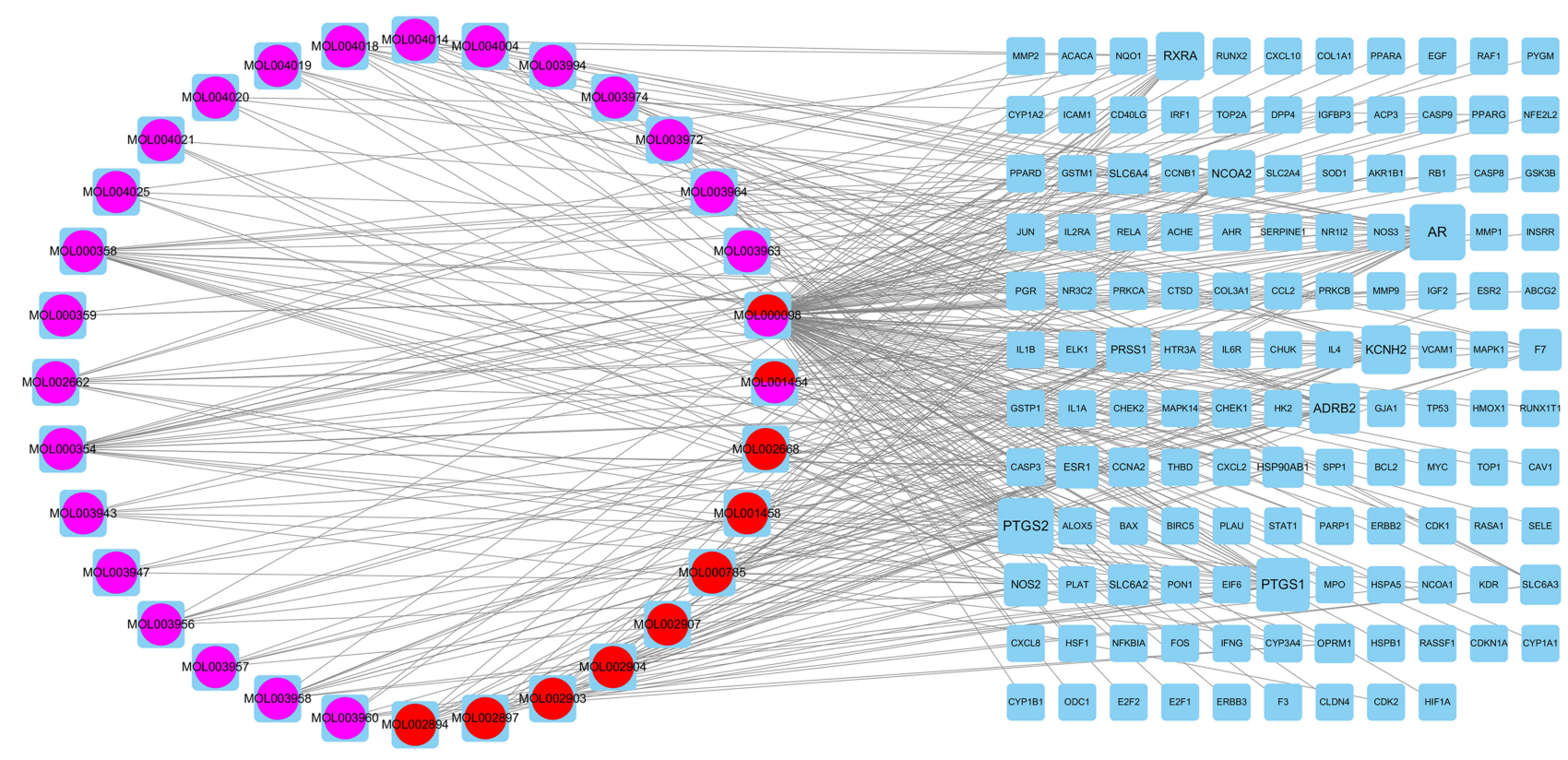

Figure 3 The C-D-T network. The pink circle represents compounds present in EF, the red circle represents compounds present in RC, and the pink and red circles represent the common compounds present in both EF and RC. 

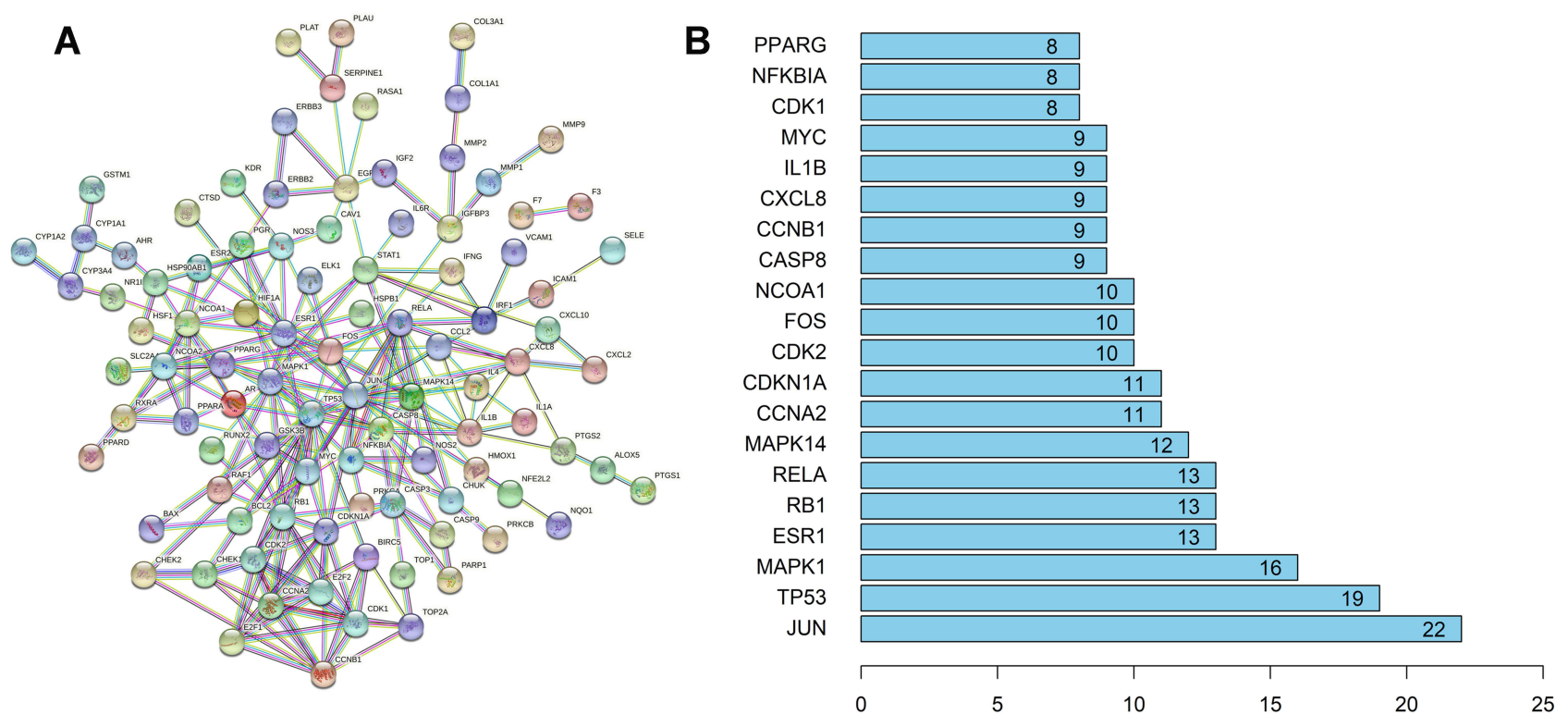

Figure 4 (A) The PPI network. (B) Bar plot showing the top 20 proteins in the PPI network. The number of proteins neighboring the target protein is plotted on the x-axis; the $y$-axis represents the target protein.
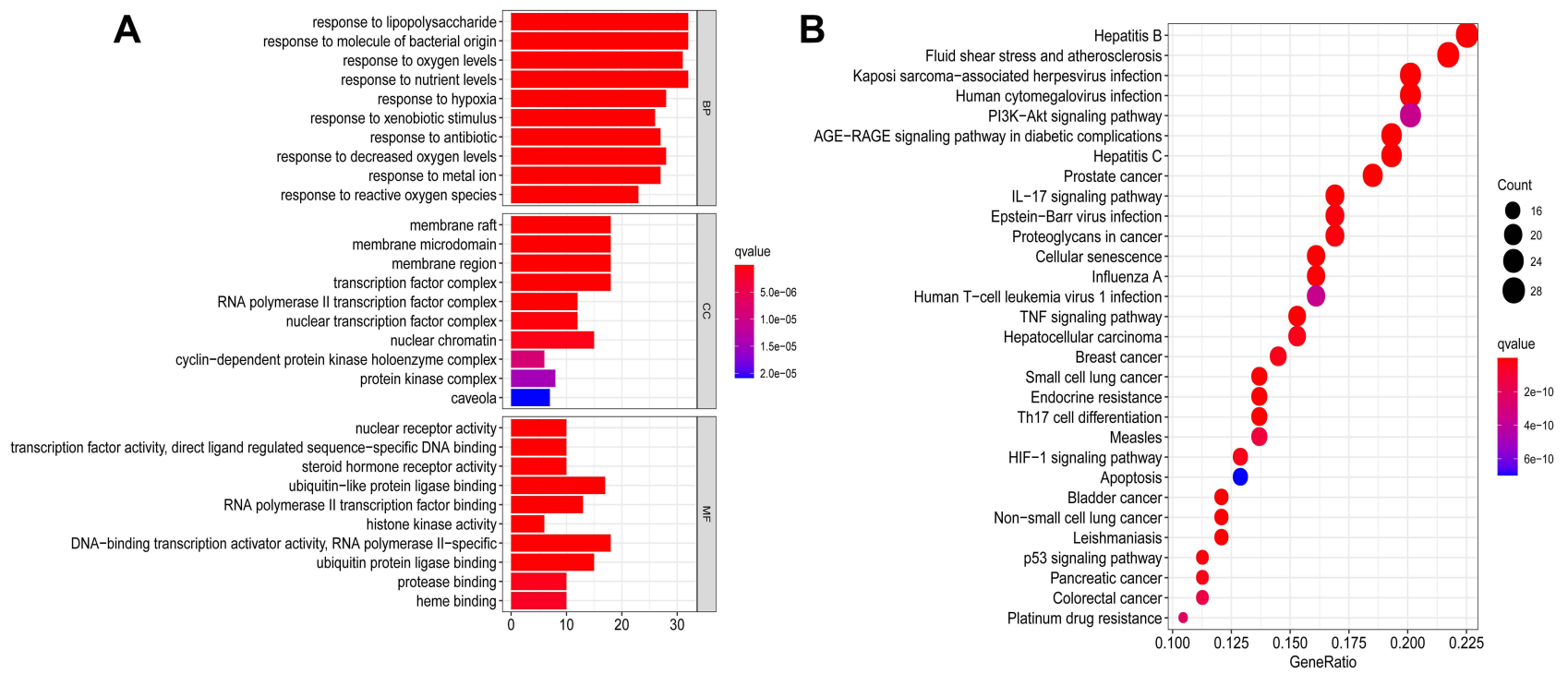

Figure 5 (A) GO analyses of the 130 potential targets associated with pancreatic cancer. The number of significantly enriched genes related to these terms is plotted on the $x$-axis; the BP, CC, and MF terms $(p<0.05)$ are plotted on the $y$-axis. (B) Dot plot showing the top 30 enriched KEGG pathways. The size of each dot corresponds to the number of genes annotated for the term, and the color of the dot corresponds to the corrected $p$-value.

smaller is its q-value; a smaller q-value indicates higher credibility and greater importance.

\section{KEGG Enrichment and C-P-T Network Analysis}

KEGG pathway enrichment analysis of the 130 genes was performed to further identify the potential pathways involved in the anti-pancreatic cancer effects of ZJP ( $p<$ $0.05)$. A total of 151 signaling pathways were acquired; the top 30 pathways, which were ranked according to their q-values, are depicted in Figure 5B. The redder the color of a pathway is, the smaller is its q-value; smaller q-values indicate higher credibility and greater importance. The results distinctly suggest that the effects of ZJP against pancreatic cancer are closely related to the PI3K-AKT signaling pathway (hsa04151), the IL-17 signaling pathway (hsa04657), the TNF signaling pathway (hsa04668), the HIF-1 signaling pathway (hsa04066), and the P53 signaling pathway (hsa04115). Among these related pathways, the 
PI3K-AKT signaling pathway, which had the highest number of enriched genes, may play a more important role in the treatment of pancreatic cancer, as shown in Figure 6.

To clarify the relationships among compounds, potential targets, and signaling pathways, a C-P-T network was constructed. The network, which is shown in Figure 7, consists of 162 nodes and 860 edges, indicating that single compounds affect multiple targets and are involved in multiple pathways and that multiple compounds are associated with individual targets and individual pathways. In brief, the anti-pancreatic cancer effects of ZJP are mediated by multiple compounds, targets, and pathways.

\section{ZJP Inhibits the Proliferation of Pancreatic Cancer Cells}

To verify the antiproliferative effect of ZJP in pancreatic cancer that was predicted by network pharmacological analysis, we assessed the functions of Panc-1 and BxPC3 cells. As the concentration of ZJP was gradually increased from 100 to $900 \mu \mathrm{g} / \mathrm{mL}$, the survival of pancreatic cancer cells decreased with dose and exposure time (24 $\mathrm{h}, 48 \mathrm{~h}$ and $72 \mathrm{~h}$ ) (Figure 8A). The half-maximal inhibitory concentration $\left(\mathrm{IC}_{50}\right)$ values for $\mathrm{ZJP}$ in various pancreatic cancer cell lines are shown in Table 2. Panc-1 and BxPC-3 cells were used in other functional assays. As shown in Figure 8B, colony formation by Panc- 1 and BxPC-3 cells was significantly reduced when the cells were treated with ZJP at 200 and $400 \mu \mathrm{g} / \mathrm{mL}$, respectively, for 3 days, suggesting that ZJP has a significant inhibitory effect on the proliferation of pancreatic cancer cells. The cell cycle analysis results further confirmed that the $\mathrm{G} 1 / \mathrm{S}$ transition is delayed in Panc-1 and BxPC-3 cells after ZJP treatment (Figure $8 \mathrm{C}$ ). The above results indicate that ZJP can significantly inhibit the proliferation of pancreatic cancer cells in vitro.

\section{ZJP Promotes the Apoptosis of Pancreatic Cancer Cells}

The effect of ZJP on the apoptosis of Panc-1 and BxPC-3 cells was measured by flow cytometry. ZJP (0, 200, 400, and $600 \mu \mathrm{g} / \mathrm{mL}$ ) was added to the cells for $48 \mathrm{~h}$. The pancreatic cancer cells were then stained with annexin V-FITC and PI to determine the degree of apoptosis. As shown in Figure 9, the percentage of apoptotic cells in the cultures increased significantly in a dose-dependent

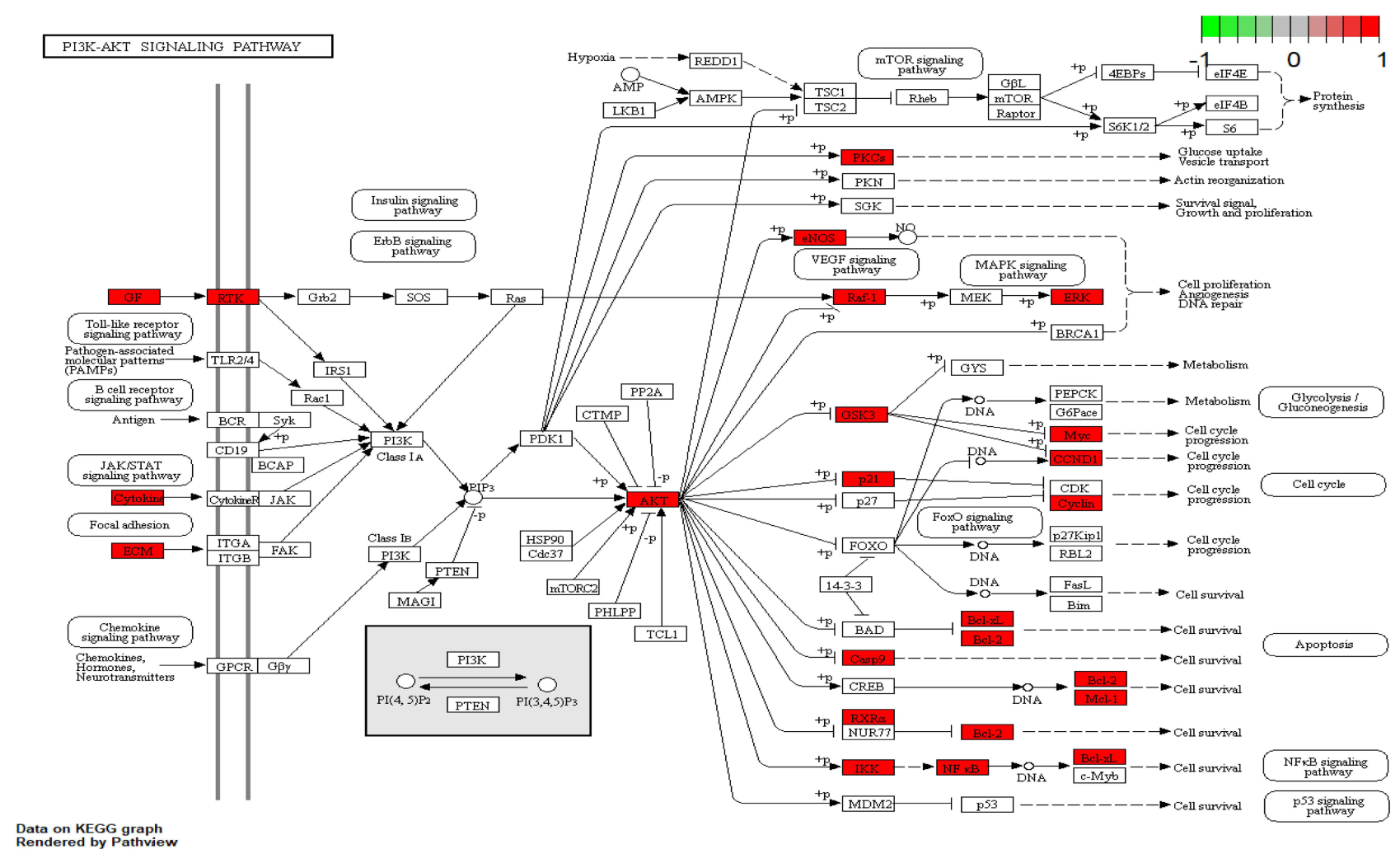

Figure 6 The mechanism through which ZJP acts against pancreatic cancer. The PI3K-AKT signaling pathway may play an important role in the treatment of pancreatic cancer. 


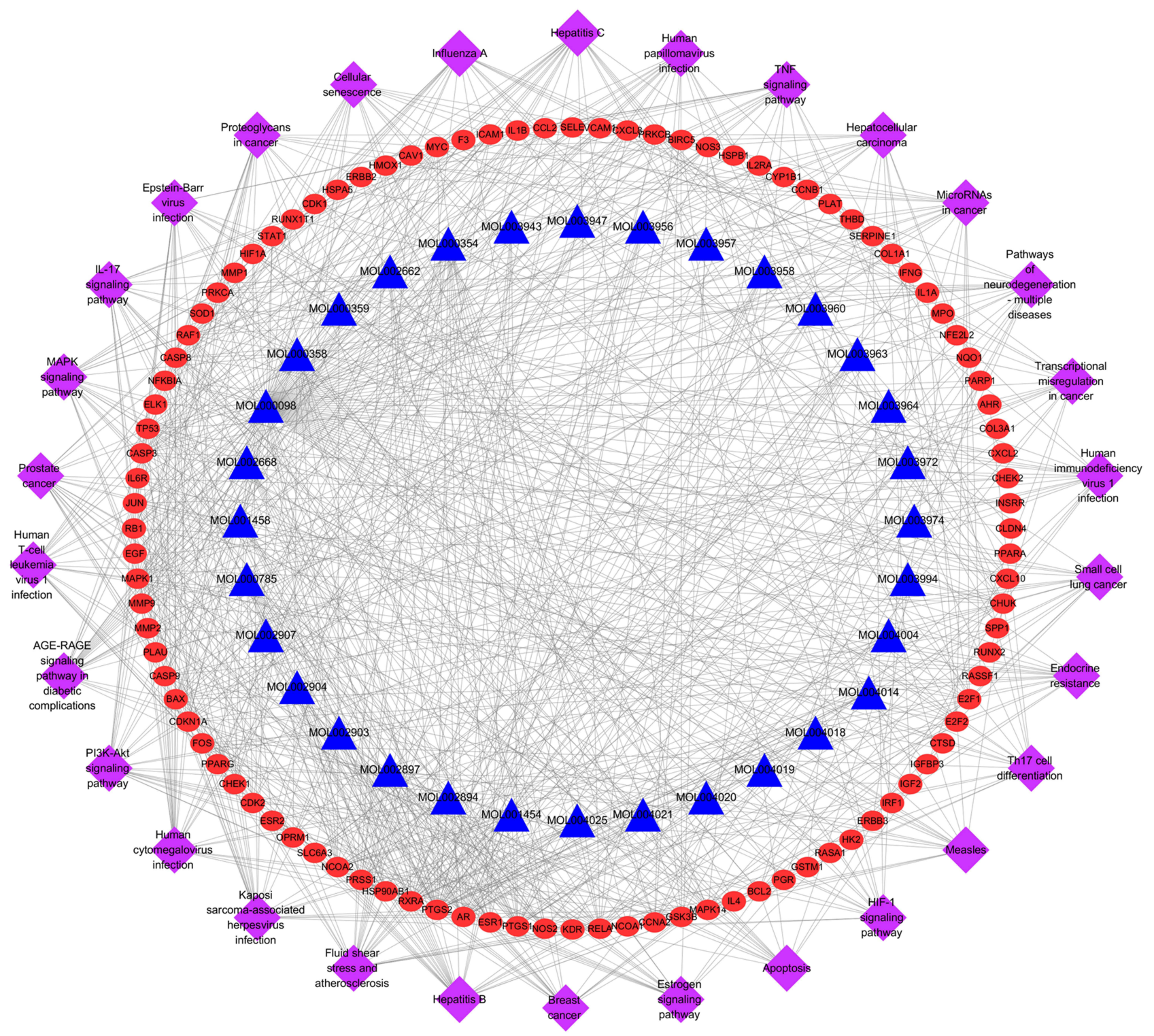

Figure 7 The C-P-T network of top 30 pathways. The red circle nodes represent genes, the blue triangle nodes represent compounds present in ZJP, and the purple diamonds represent pathways.

manner after ZJP treatment, strongly suggesting that ZJP induces apoptosis of Panc-1 and BxPC-3 cells.

\section{ZJP Inhibits the EGFR/PI3K/AKT Pathway in Pancreatic Cancer Cells}

We further investigated the molecular mechanism through which ZJP affects apoptosis in Panc-1 and BXPC-3 cells. The EGFR/PI3K/AKT pathway, a key pathway related to the anti-pancreatic cancer effect of ZJP, was analyzed by Western blotting. As shown in Figure 10, EGFR, PI3K and AKT protein levels were significantly reduced in a dose-dependent manner after treatment of the cells with ZJP. In contrast, the protein levels of BAD, caspase 9 and caspase 3 were significantly increased. The results show that ZJP induced the apoptosis of Panc-1 and BxPC-3 cells through the EGFR/PI3K/ AKT/caspase pathway, thereby achieving an antitumor effect.

\section{Discussion}

Pancreatic cancer is a common malignancy of the digestive tract. ${ }^{32,33}$ In recent years, the antitumor effects of TCMs have received increasing attention because of their clear pharmacological effects and relatively low number of side effects. A large number of patients choose natural therapy with TCM because TCMs are considered to be multicomponent, 
A

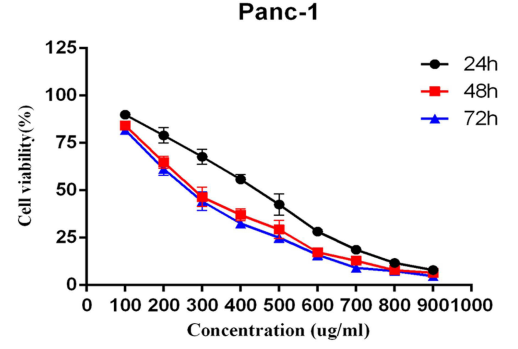

BxPC-3

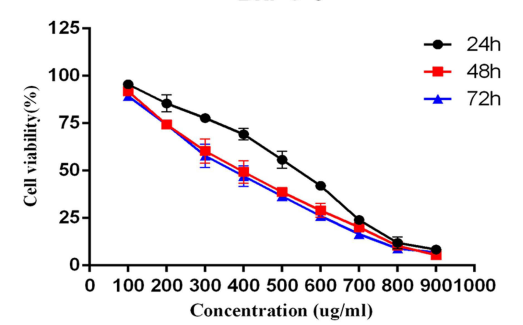

B
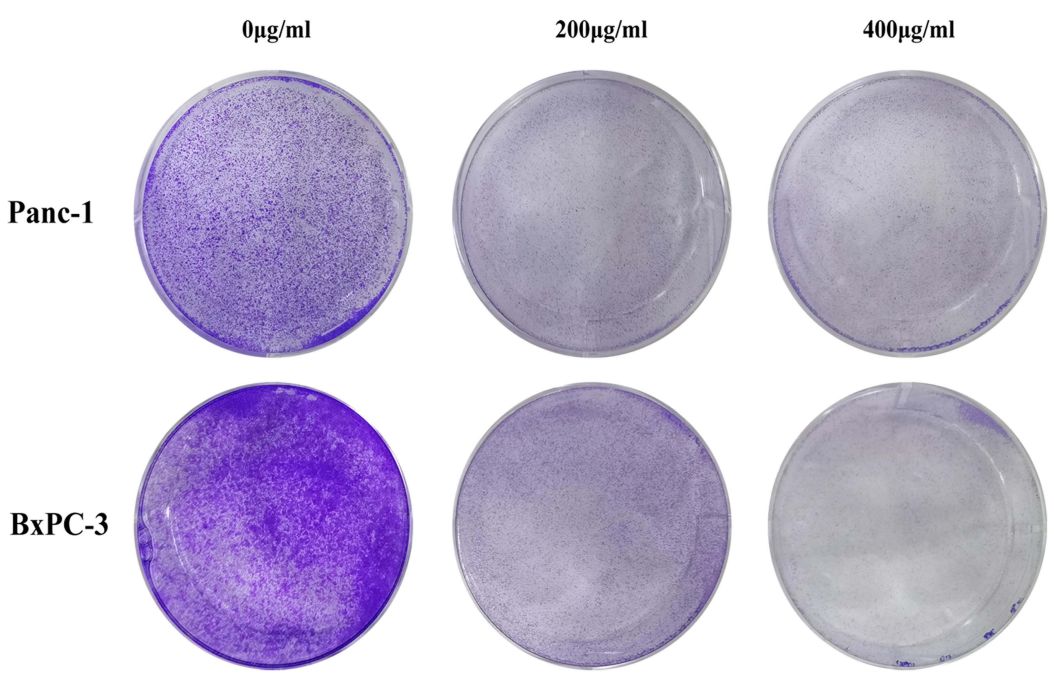

C
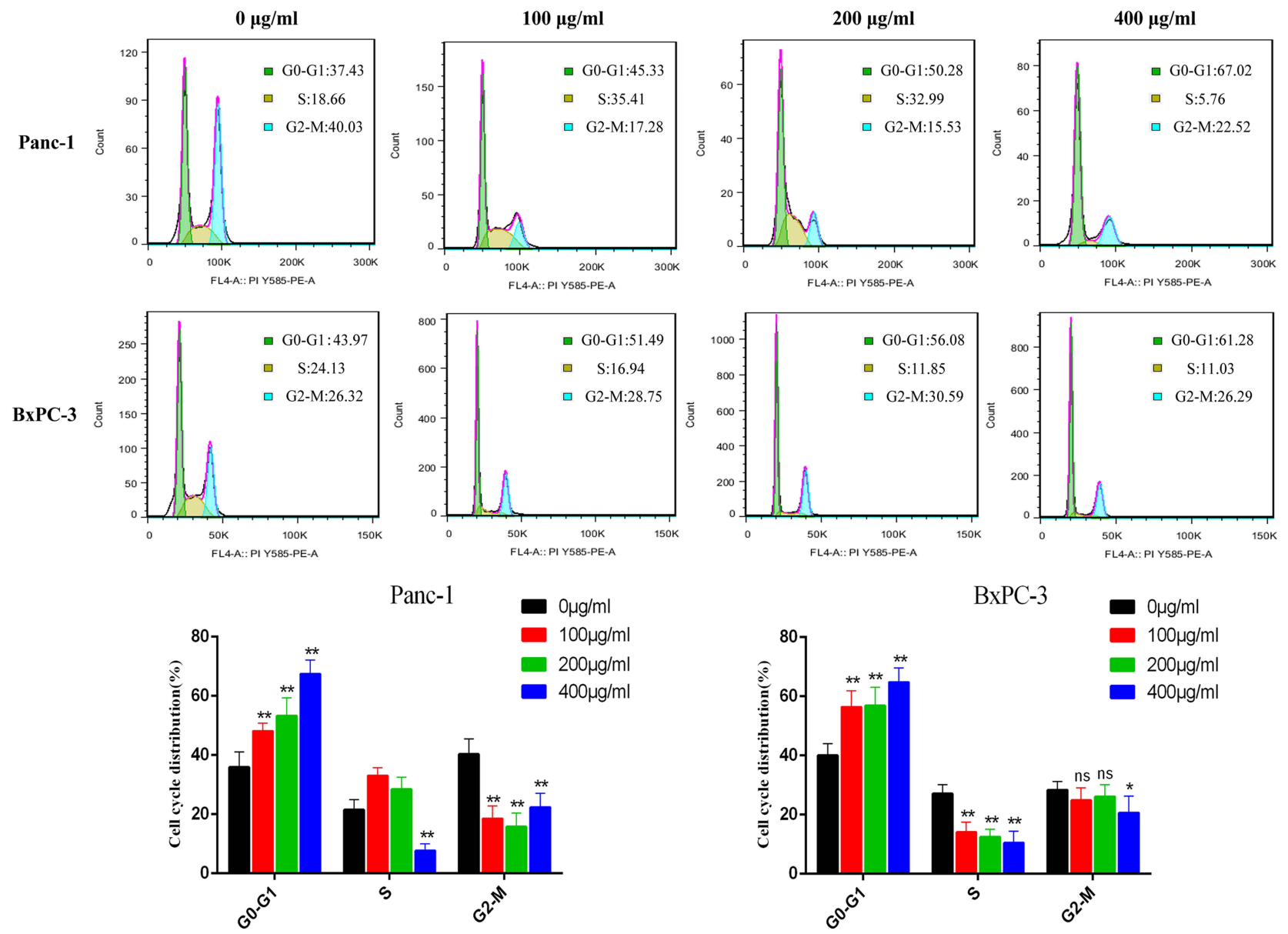

Figure 8 ZJP inhibits the proliferation of pancreatic cancer cells. (A) ZJP decreases the viability of pancreatic cancer cells as measured by the CCK-8 assay. Pancreatic cancer cells were treated with various concentrations of ZJP for 24, 48, and $72 \mathrm{~h}$. (B) Representative images showing colonies formed by pancreatic cancer cells treated with various concentrations of ZJP for 3 days. (C) Representative images and statistical graphs of Panc-I and BxPC-3 cell cycle analysis. *P $<0.05$, **P $<0.0 \mathrm{I}$ versus the untreated group. 
Table 2 The $I_{50}$ Values of Pancreatic Cancer Cells Treated with ZJP $(\mu \mathrm{g} / \mathrm{mL})$

\begin{tabular}{|l|c|c|c|}
\hline Cell Lines & $\mathbf{2 4 h}$ & $\mathbf{4 8 h}$ & $\mathbf{7 2 h}$ \\
\hline Panc-I & 338.2 & 262.3 & 254.6 \\
BxPC-3 & 661.4 & 308.8 & 299.3 \\
\hline
\end{tabular}

multitarget and multistage medicines. Chinese medicine includes a large number of bioactive ingredients, some of which have anti-pancreatic cancer activity. ${ }^{2}$ In 2007, three new herbal medicines derived from Chinese medicine, ixabepilone, trabectedin and temsirolimus, were approved, prompting the consideration of TCM as a source of innovative targeted anticancer therapies. ${ }^{34}$
Chinese medicine has been practiced for thousands of years and is now widely recognized as an alternative method for treating cancer. $^{35}$ A large number of natural products derived from Chinese herbal medicines have antitumor activities, including antiproliferative, proapoptotic, antimetastatic, and antiangiogenic activities, and these medicines can also regulate autophagy, reverse multidrug resistance, maintain immune homeostasis, and enhance the effects of chemotherapy. ${ }^{36}$ The signaling pathways that control cell proliferation, invasion, metastasis and angiogenesis are often dysregulated during the pathological progression of tumors. From a therapeutic point of view, these signaling pathways have become an important source of targets for tumor therapy. ${ }^{37}$ According to drug
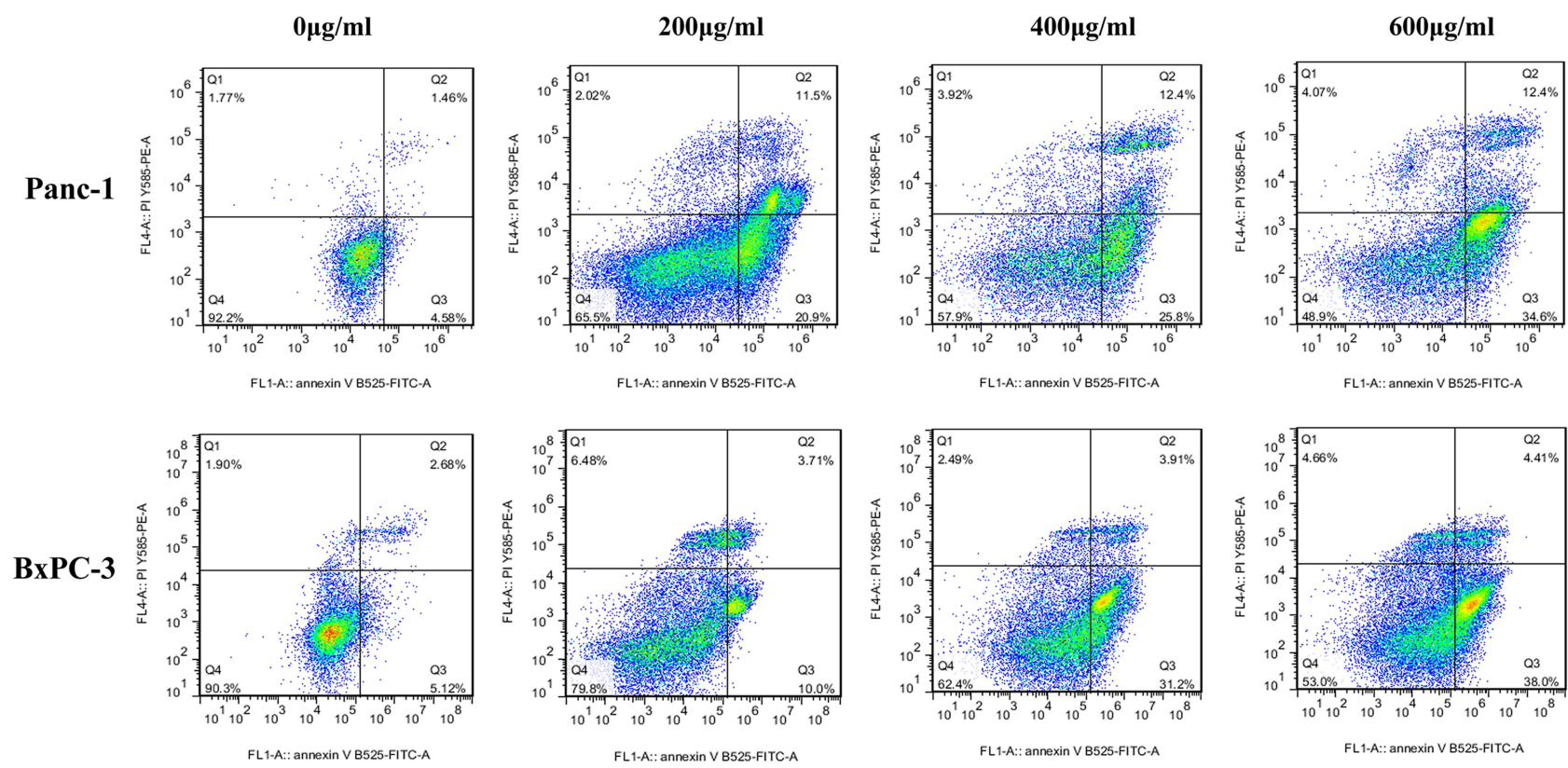

NC $200 \mu \mathrm{g} / \mathrm{ml}$

$400 \mu \mathrm{g} / \mathrm{ml}$

$600 \mu \mathrm{g} / \mathrm{ml}$

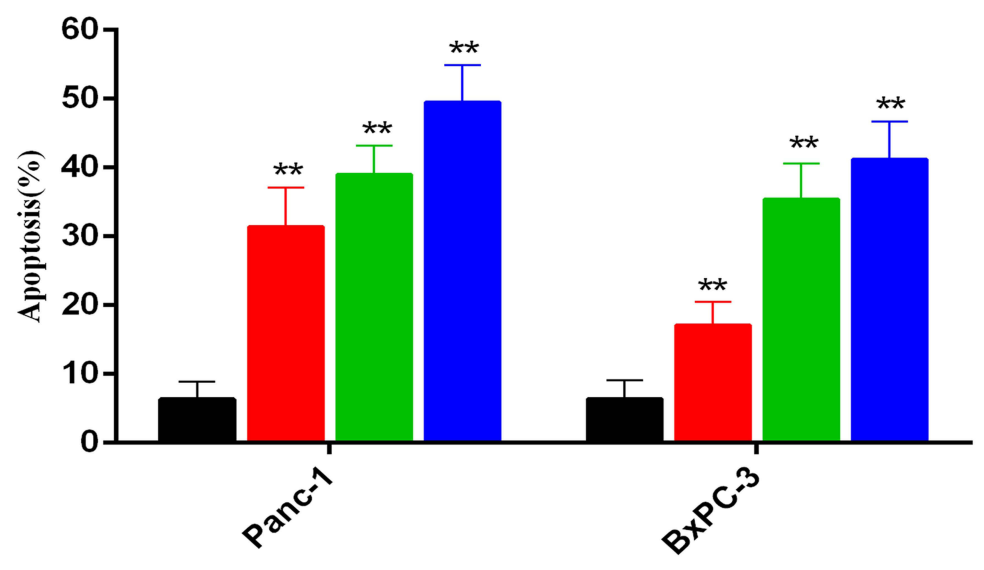

Figure 9 Pancreatic cancer cells were treated with various concentrations of ZJP, and apoptosis of the cells was then analyzed by flow cytometry after annexin V-FITC/PI staining. The percentage of apoptotic cells is presented as the mean \pm SD of three independent experiments; **P $<0.01$ versus the untreated group. 
A

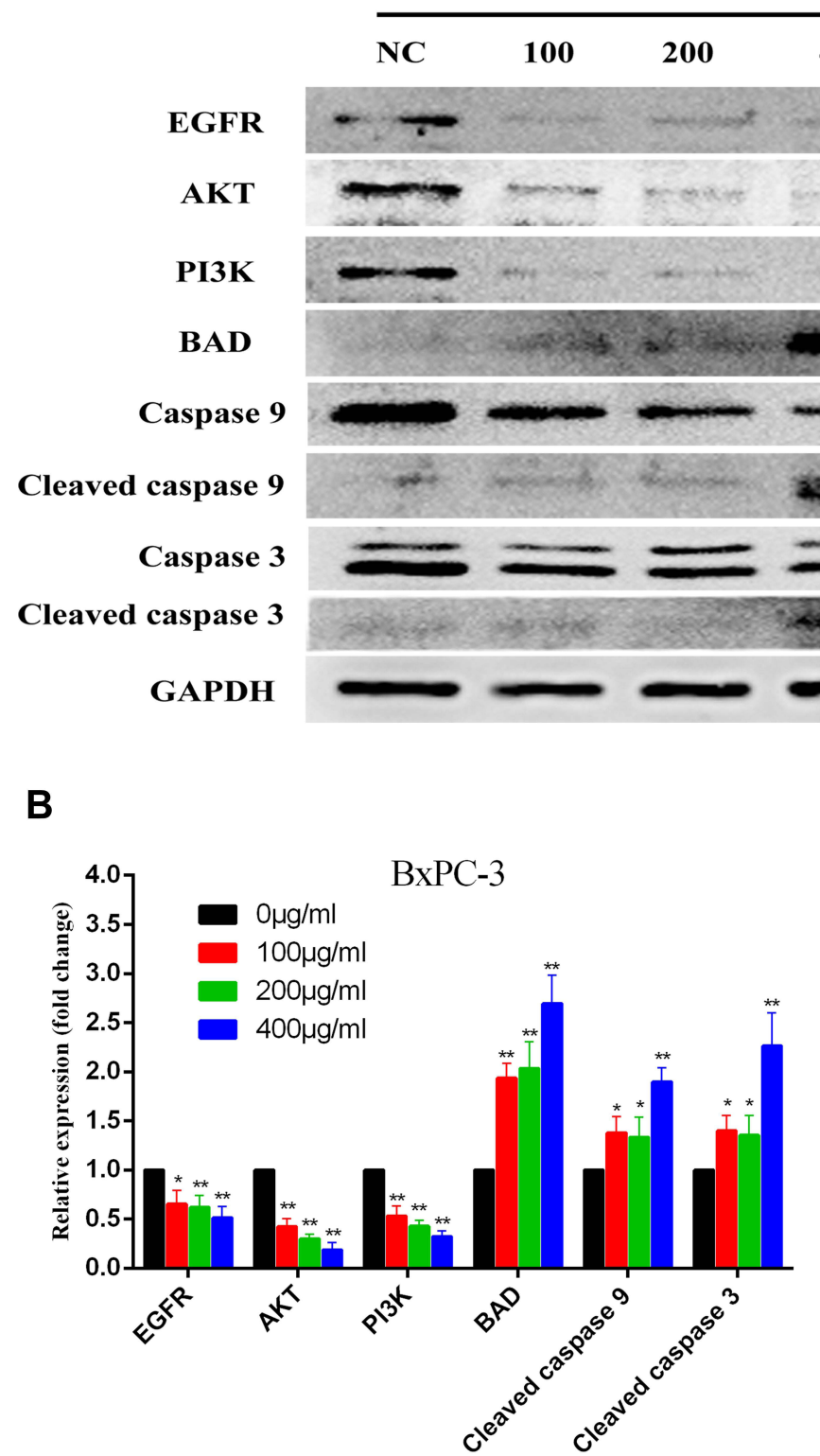

Panc-1

400

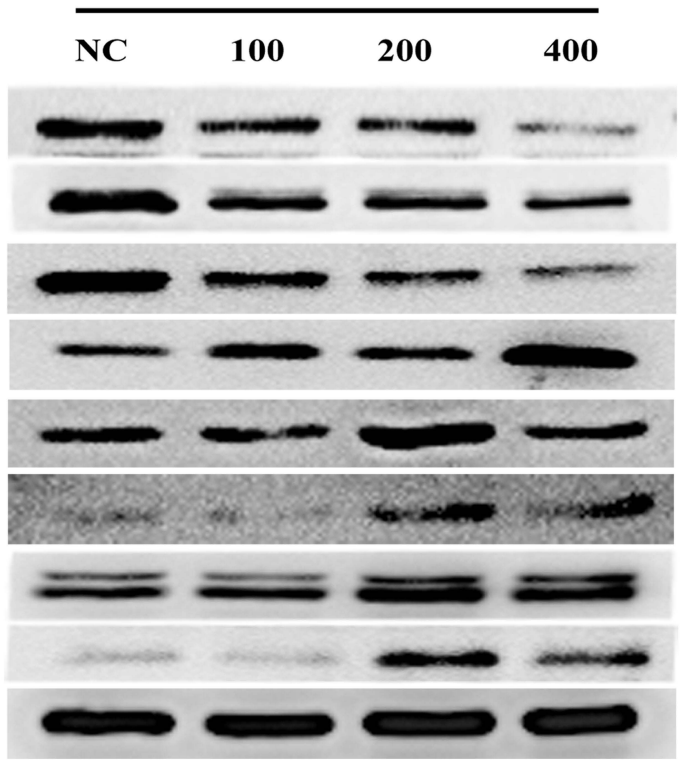

Figure 10 Relative expression of proteins related to ZJP treatment in pancreatic cancer cells. (A) Western Blotting revealed the changes of related proteins in BxPC-3 and Panc-I cell lines after ZJP treatment; (B) Western Blotting statistical analysis showed that the expression levels of EGFR, AKT, and PI3K were down-regulated. Cleaved caspase $3 / 9$ and $B A D$ were up-regulated. $* \mathrm{P}<0.05$, $* * \mathrm{P}<0.01$ versus the untreated group.

target prediction and comprehensive pathway analysis, ZJP may play an important antitumor role by regulating cell proliferation and survival. ${ }^{38}$ As predicted by network pharmacological methods, ZJP may play a therapeutic role in tumors mainly by regulating tumor cell proliferation and cell survival through the PI3K-NF- $\kappa$ B, EGFR-MAPK, and CCND1 signaling pathways. Guo et al showed that ZJP significantly reduced the viability and colony-forming ability of hepatocellular carcinoma (HCC) cells, inhibited cell activity in G0/G1 phase, and inhibited cell migration and invasion in a dose-dependent manner. ${ }^{19}$ The authors

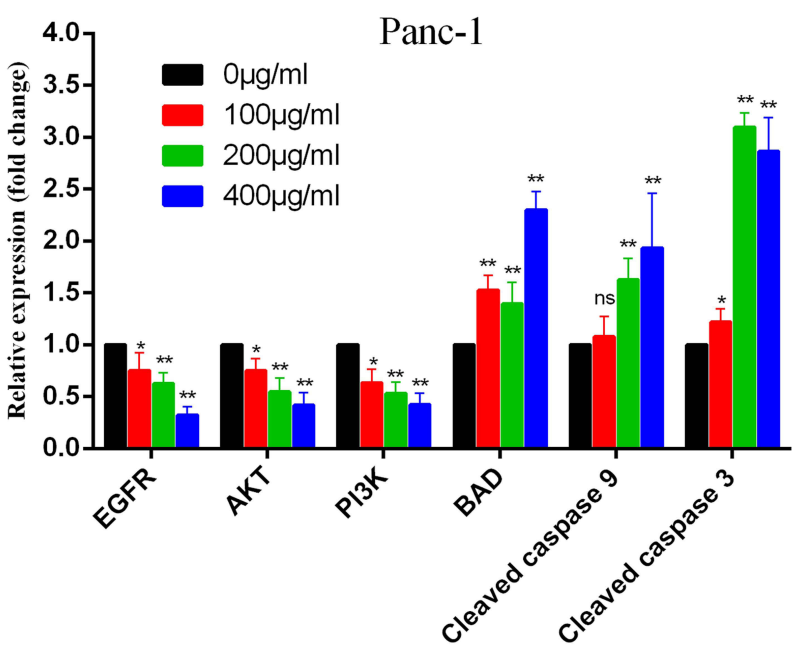

suggest that the high concentration of compounds in the compound target network may be the main factor that accounts for the effect of ZJP in the treatment of HCC. In addition, quercetin, a natural flavonoid, is an important component of ZJP, and it exerts anticancer effects by inhibiting the PI3K pathway. $^{39}$ Furthermore, quercetin promotes cell death and enhances chemotherapy sensitivity in human pancreatic cancer cells through the RAGE/ PI3K/AKT/mTOR axis. ${ }^{40}$ Berberine is also an important component of ZJP, and recent cell metabolomics studies have revealed that berberine decreases pancreatic cancer 
cell viability and inhibits metastasis by regulating citric acid metabolism. ${ }^{41}$ Furthermore, liposomes delivering both berberine and irinotecan were more effective and exhibited lower intestinal toxicity in the treatment of pancreatic cancer than liposomes delivering either component alone. ${ }^{42}$ All of the above studies support our network pharmacological prediction and prove the feasibility of network pharmacological methods in the study of the mechanism of action of TCM.

Based on network pharmacology, bioinformatics analysis and in vitro experiments, this study revealed the bioactive compounds and molecular mechanisms through which ZJP works in the treatment of pancreatic cancer. Previous studies have shown that ZJP mainly inhibits proliferation, induces cell apoptosis and autophagic cell death, and has certain anticancer effects on colon cancer, breast cancer, liver cancer, lung cancer, colorectal cancer, and other types of cancer. ${ }^{27}$ In the current study, a total of 294 types of Chinese herbal medicines were identified, and 41 components were followed up using comprehensive retrieval methods in the Chinese herbal medicine database and the BATMAN-TCM database. In addition, 2969 pancreatic cancer-related genes were identified. In a comparison of the 173 targets of ZJP with the 2969 pancreatic cancer-related genes, 130 common targets were obtained. The results of PPI analysis showed that Jun, TP53 and MAPK1 might be the most important targets of ZJP in pancreatic cancer. GO enrichment analysis confirmed the biological functions of the 130 target genes of ZJP against pancreatic cancer. Moreover, through the KEGG pathway enrichment analysis of 130 genes, 151 related signaling pathways were obtained; among these, the PI3K-AKT signaling pathway may play the most important role in the treatment of pancreatic cancer. The KEGG results showed that among tumor-related signaling pathways, the PI3K-AKT signaling pathway displayed the highest degree of enrichment, suggesting that it plays a key role in the antimetastatic effect of ZJP in pancreatic cancer. The AKT family plays an important role in the regulation of biological functions such as cell survival, proliferation, metabolism and growth and affects tumor growth. AKT is involved in the treatment of a variety of cancers through multiple signaling pathways, especially the PI3K-AKT signaling pathway. Increasing evidence shows that the PI3K/AKT signaling pathway is involved in the cell cycle, cell proliferation, cell growth, cell migration, angiogenesis and apoptosis and that it is one of the most common signal transduction pathways involved in cancer. ${ }^{43-45}$ In short, the anti-pancreatic cancer effect of ZJP is achieved through multiple compounds, multiple targets and multiple pathways. Moreover, in vitro experiments verified that ZJP inhibits the proliferation and induces the apoptosis of pancreatic cancer cells by inhibiting the PI3K-AKT signaling pathway. In addition, EGFR is a transmembrane glycoprotein that is a member of the protein kinase superfamily and acts as a receptor for members of the epidermal growth factor family. ${ }^{46}$ The binding of this protein to ligands triggers its tyrosine kinase activity, leading to cell proliferation. In pancreatic cancer, overexpression of EGFR plays an important role in the regulation of tumor cell proliferation, angiogenesis, invasion and metastasis. ${ }^{47,48}$ In vitro experiments showed that after ZJP treatment of pancreatic cancer cells, EGFR expression was downregulated, the cell cycle transition from $\mathrm{G} 1$ to $\mathrm{S}$ phase was inhibited, and the proliferation of the cells was inhibited. Thus, according to experimental verification, ZJP treatment can effectively block the EGFR, and this contributes to the treatment of pancreatic cancer.

In future research, we should make full use of recent technological progress to further improve the isolation and identification of TCM compounds and clarify their mechanisms of action, potential toxicities and adverse reactions. This could improve the efficacy of promising TCM compounds in the treatment of pancreatic cancer.

\section{Conclusion}

In this study, we utilized network pharmacological prediction methods, bioinformatics analysis and in vitro experiments to identify and verify the active compounds of ZJP and to delineate their mechanisms in pancreatic cancer. We demonstrated that ZJP plays a key role in inhibiting cell proliferation and promoting apoptosis by activating its target to combat pancreatic cancer. This study provides a promising multidisciplinary approach for characterizing TCM compounds for the treatment of diseases.

\section{Abbreviations}

AKT, serine-threonine kinase; BAD, BCL2 associated agonist of cell death; BPs, biological processes; CCND1, cyclin D1; C-D-T, Herb-disease-target; C-P-T, compoundpathway-target; CCs, Cell components; DL, druglikeness; DMSO, dimethyl sulfoxide; EF, evodiae fructus; EGFR, epidermal growth factor receptor; GO, gene ontology; HIF-1, hypoxia-inducible factor 1; HCC, Hepatocellular carcinoma; IL-17, Interleukin 17; $\mathrm{IC}_{50}$, 
$50 \%$ inhibitory concentration; JUN, jun proto-oncogene; KEGG, Kyoto Encyclopedia of Genes and Genomes; MFs, molecular functions; MAPK1, Mitogen-activated protein kinase 1; NF- $\kappa \mathrm{B}$, Nuclear factor kappa B; OB, oral bioavailability; OMIM, Online Mendelian Inheritance in Man; PPI, Protein-protein interaction; PI3K, Phosphatidylinositol 3-kinase; RC, Rhizoma Coptidis; TCM, Traditional Chinese medicine; TCMSP, Traditional Chinese Medicines for Systems Pharmacology; TNF, tumor necrosis factor; TP53, tumor protein p53; ZJP, Zuojin pills.

\section{Acknowledgments}

This study was supported by the Zhejiang Province Public Welfare Technology Application Research Project (LGF21H160022); Zhejiang Medical and Health Science and Technology Program (2017KY711); and Project of Taizhou Central Hospital (2019KT003).

\section{Disclosure}

The authors report no conflicts of interest in this work.

\section{References}

1. Goral V. Pancreatic cancer: pathogenesis and diagnosis. Asian Pac $J$ Cancer Prev. 2015;16(14):5619-5624. doi:10.7314/ APJCP.2015.16.14.5619

2. Gao Y, Chen S, Sun J, et al. Traditional Chinese medicine may be further explored as candidate drugs for pancreatic cancer: a review. Phytother Res. 2021;35(2):603-628.

3. Kong F, Liu X, Zhou Y, et al. Downregulation of METTL14 increases apoptosis and autophagy induced by cisplatin in pancreatic cancer cells. Int J Biochem Cell Biol. 2020;122:105731.

4. Hou X, Yang L, Wang K, et al. HELLS, a chromatin remodeler is highly expressed in pancreatic cancer and downregulation of it impairs tumor growth and sensitizes to cisplatin by reexpressing the tumor suppressor TGFBR3. Cancer Med. 2021;10(1):350-364. doi:10.1002/cam4.3627

5. Sung H, Ferlay J, Siegel RL, et al. Global cancer statistics 2020: GLOBOCAN estimates of incidence and mortality worldwide for 36 cancers in 185 countries. CA Cancer J Clin. 2021;71(3):209-249.

6. McGuigan A, Kelly P, Turkington RC, Jones C, Coleman HG, McCain RS. Pancreatic cancer: a review of clinical diagnosis, epidemiology, treatment and outcomes. World J Gastroenterol. 2018;24 (43):4846-4861. doi:10.3748/wjg.v24.i43.4846

7. Zhu H, Li T, Du Y, Li M. Pancreatic cancer: challenges and opportunities. BMC Med. 2018;16:214.

8. Mizrahi JD, Surana R, Valle JW, Shroff RT. Pancreatic cancer. Lancet. 2020;395(10242):2008-2020. doi:10.1016/S0140-6736(20) 30974-0

9. Bednar F, Simeone DM. Recent advances in pancreatic surgery. Curr Opin Gastroenterol. 2014;30(5):518-523. doi:10.1097/MOG.000 0000000000096

10. Bettegowda C, Sausen M, Leary RJ, et al. Detection of circulating tumor DNA in early- and late-stage human malignancies. Sci Transt Med. 2014;6(224):224ra24-224ra24. doi:10.1126/scitranslmed.3 007094
11. Yao C-L, Zhang J-Q, Li J-Y, Wei W-L, Wu S-F, Guo D-A. Traditional Chinese medicine (TCM) as a source of new anticancer drugs. Nat Prod Rep. 2021. doi:10.1039/D0NP00057D

12. Kim K-C, Yook J-H, Eisenbraun J, Kim B-S, Huber R. Quality of life, immunomodulation and safety of adjuvant mistletoe treatment in patients with gastric carcinoma - a randomized, controlled pilot study. BMC Complement Altern Med. 2012;12(1). doi:10.1186/ 1472-6882-12-172

13. Gavaraskar K, Dhulap S, Hirwani RR. Therapeutic and cosmetic applications of evodiamine and its derivatives - a patent review. Fitoterapia. 2015;106:22-35. doi:10.1016/j.fitote.2015.07.019

14. Chen G. Effects of shenfu injection on chemotherapy-induced adverse effects and quality of life in patients with advanced nonsmall cell lung cancer: a systematic review and meta-analysis. J Cancer Res Ther. 2018;14(10):S549-S555. doi:10.4103/0973-1482.187299

15. Su X-L, Wang J-W, Che H, et al. Clinical application and mechanism of traditional Chinese medicine in treatment of lung cancer. Chin Med J. 2020;133(24):2987-2997. doi:10.1097/CM9.000000000 0001141

16. Huang S, Peng W, Mao D, et al. Kangai injection, a traditional Chinese medicine, improves efficacy and reduces toxicity of chemotherapy in advanced colorectal cancer patients: a systematic review and meta-analysis. Evid Based Complement Alternat Med. 2019;2019:1-15. doi:10.1155/2019/8423037

17. Mao D, Feng L, Huang S, Zhang S, Peng W, Zhang S. Meta-analysis of xihuang pill efficacy when combined with chemotherapy for treatment of breast cancer. Evid Based Complement Alternat Med. 2019;2019:1-14. doi:10.1155/2019/3502460

18. Wang Q, Jiao L, Wang S, et al. Maintenance chemotherapy with Chinese herb medicine formulas vs. with placebo in patients with advanced non-small cell lung cancer after first-line chemotherapy: a multicenter, randomized, double-blind trial. Front Pharmacol. 2018;9:1233. doi:10.3389/fphar.2018.01233

19. Guo W, Huang J, Wang N, et al. Integrating network pharmacology and pharmacological evaluation for deciphering the action mechanism of herbal formula zuojin pill in suppressing hepatocellular carcinoma. Front Pharmacol. 2019;10:1185. doi:10.3389/fphar.2019.01185

20. Tong Y, Wang R, Liu X, et al. Zuojin pill ameliorates chronic atrophic gastritis induced by MNNG through TGF- $\beta 1 / \mathrm{PI} 3 \mathrm{~K} / \mathrm{Akt}$ axis. $J$ Ethnopharmacol. 2021;271:113893. doi:10.1016/j. jep.2021.113893

21. Pan J, Xu Y, Song H, Zhou X, Yao Z, Ji G. Extracts of Zuo Jin Wan, a traditional Chinese medicine, phenocopies 5-HTR1D antagonist in attenuating Wnt/beta-catenin signaling in colorectal cancer cells. BMC Complement Altern Med. 2017;17:1-11.

22. Wang N, Tan H-Y, Li L, Yuen M-F, Feng Y. Berberine and coptidis rhizoma as potential anticancer agents: recent updates and future perspectives. $J$ Ethnopharmacol. 2015;176:35-48. doi:10.1016/j. jep.2015.10.028

23. Lam P, Cheung F, Tan HY, Wang N, Yuen MF, Feng Y. Hepatoprotective effects of Chinese medicinal herbs: a focus on anti-inflammatory and anti-oxidative activities. Int $J$ Mol Sci. 2016;17(4):465. doi:10.3390/ijms17040465

24. Xu S, Peng J, Li Y, et al. Pharmacokinetic comparisons of rutaecarpine and evodiamine after oral administration of $\mathrm{Wu}-\mathrm{Chu}-\mathrm{Yu}$ extracts with different purities to rats. J Ethnopharmacol. 2012;139 (2):395-400. doi:10.1016/j.jep.2011.11.023

25. Chou S-T, Hsiang C-Y, Lo H-Y, et al. Exploration of anti-cancer effects and mechanisms of Zuo-Jin-Wan and its alkaloid components in vitro and in orthotopic HepG2 xenograft immunocompetent mice. BMC Complement Altern Med. 2017;17(1):1-11. doi:10.1186/ s12906-017-1586-6

26. Du J, Sun Y, Wang X-F, Lu -Y-Y, Zhou Q-M, Su S-B. Establishment of an experimental breast cancer ZHENG model and curative effect evaluation of Zuo-Jin Wan. Evid Based Complement Alternat Med. 2013;2013:1-6. doi:10.1155/2013/324732 
27. Huang S, Zhang Z, Li W, et al. Network pharmacology-based prediction and verification of the active ingredients and potential targets of zuojinwan for treating colorectal cancer. Drug Des Devel Ther. 2020;14:2725-2740. doi:10.2147/DDDT.S250991

28. Chen Y, Kern TS, Kiser PD, Palczewski K. Eyes on systems pharmacology. Pharmacol Res. 2016;114:39-41. doi:10.1016/j. phrs.2016.09.026

29. Jing C, Sun Z, Xie X, et al. Network pharmacology-based identification of the key mechanism of Qinghuo Rougan formula acting on uveitis. Biomed Pharmacother. 2019;120:109381. doi:10.1016/j. biopha.2019.109381

30. Li S, Zhang B. Traditional Chinese medicine network pharmacology: theory, methodology and application. Chin J Nat Med. 2013;11 (2):110-120. doi:10.1016/S1875-5364(13)60037-0

31. Yang S, Zhang J, Yan Y, et al. Network pharmacology-based strategy to investigate the pharmacologic mechanisms of atractylodes macrocephala Koidz. for the treatment of chronic gastritis. Front Pharmacol. 2019;10:1629.

32. Luo Y, Hu J, Liu Y, et al. Invadopodia: a potential target for pancreatic cancer therapy. Crit Rev Oncol Hematol. 2021;159:103236. doi:10.1016/j.critrevonc.2021.103236

33. Martinez-Useros J, Martin-Galan M, Garcia-Foncillas J. The match between molecular subtypes, histology and microenvironment of pancreatic cancer and its relevance for chemoresistance. Cancers. 2021;13(2):322. doi:10.3390/cancers 13020322

34. Nie J, Zhao C, Deng L, et al. Efficacy of traditional Chinese medicine in treating cancer (review). Biomed Rep. 2016;4(1):3-14. doi:10.3892/br.2015.537

35. Xiang Y, Guo Z, Zhu P, Chen J, Huang Y. Traditional Chinese medicine as a cancer treatment: modern perspectives of ancient but advanced science. Cancer Med. 2019;8(5):1958-1975. doi:10.1002/ cam4.2108

36. Luo H, Vong CT, Chen HB, et al. Naturally occurring anti-cancer compounds: shining from Chinese herbal medicine. Chin Med. 2019;14(1):58.

37. Moeini A, Cornella H, Villanueva A. Emerging signaling pathways in hepatocellular carcinoma. Liver Cancer. 2012;1(2):83-93. doi:10.1159/000342405

38. Tanaka S, Arii S. Molecular targeted therapies in hepatocellular carcinoma. Semin Oncol. 2012;39(4):486-492. doi:10.1053/j. seminoncol.2012.05.005
39. Maurya AK, Vinayak M. Anticarcinogenic action of quercetin by downregulation of phosphatidylinositol 3-kinase (PI3K) and protein kinase $\mathrm{C}(\mathrm{PKC})$ via induction of p53 in hepatocellular carcinoma (HepG2) cell line. Mol Biol Rep. 2015;42(9):1419-1429. doi:10.1007/s11033-015-3921-7

40. Lan CY, Chen SY, Kuo CW, Lu CC, Yen GC. Quercetin facilitates cell death and chemosensitivity through RAGE/PI3K/AKT/mTOR axis in human pancreatic cancer cells. J Food Drug Anal. 2019;27 (4):887-896. doi:10.1016/j.jfda.2019.07.001

41. Liu J, Luo X, Guo R, Jing W, Lu H. Cell metabolomics reveals berberine-inhibited pancreatic cancer cell viability and metastasis by regulating citrate metabolism. J Proteome Res. 2020;19 (9):3825-3836. doi:10.1021/acs.jproteome.0c00394

42. Wang X, Liu Y, Xu W, et al. Irinotecan and berberine co-delivery liposomes showed improved efficacy and reduced intestinal toxicity compared with Onivyde for pancreatic cancer. Drug Deliv Transl Res. 2021:1-12. doi:10.1007/s13346-020-00884-4

43. ShuoWang SZ, Song Z, Gong X, et al. Chloroform extract from sophora tonkinensis gagnep. inhibit proliferation, migration, invasion and promote apoptosis of nasopharyngeal carcinoma cells by silencing the PI3K/AKT/mTOR signaling pathway. J Ethnopharmacol. 2021;271:113879. doi:10.1016/j.jep.2021.113879

44. Yu X, Li S, Pang M, et al. TSPAN7 exerts anti-tumor effects in bladder cancer through the PTEN/PI3K/AKT pathway. Front Oncol. 2021;10: Article 613869. doi:10.3389/fonc.2020.613869

45. Zhang M, Lin H, Ge X, Xu Y. Overproduced CPSF4 promotes cell proliferation and invasion via PI3K-AKT signaling pathway in oral squamous cell carcinoma. J Oral Maxillofac Surg. 2021;79(5):1177e1.

46. Seshacharyulu P, Ponnusamy MP, Haridas D, Jain M, Ganti AK, Batra SK. Targeting the EGFR signaling pathway in cancer therapy. Expert Opin Ther Targets. 2012;16(1):15-31. doi:10.1517/ 14728222.2011 .648617

47. Qian W, Chen K, Qin T, et al. The EGFR-HSF1 axis accelerates the tumorigenesis of pancreatic cancer. J Exp Clin Cancer Res. 2021;40 (1):1-9. doi:10.1186/s13046-020-01823-4

48. Barman S, Fatima I, Gowrikumar S, Singh AB, Batra SK, Dhawan P. MASTL regulates EGFR signaling to promote pancreatic cancer progression. Pancreas. 2020;49(10):1400.

\section{Publish your work in this journal}

Drug Design, Development and Therapy is an international, peerreviewed open-access journal that spans the spectrum of drug design and development through to clinical applications. Clinical outcomes, patient safety, and programs for the development and effective, safe, and sustained use of medicines are a feature of the journal, which has also been accepted for indexing on PubMed Central. The manuscript management system is completely online and includes a very quick and fair peer-review system, which is all easy to use. Visit http://www. dovepress.com/testimonials.php to read real quotes from published authors. 